\title{
Prenatal diagnosis of congenital anomalies of genito-urinary system on fetal magnetic resonance imaging
}

\author{
Saryu Gupta ${ }^{1+} \mathbb{D}$, Jaswinder Kaur Mohi ${ }^{1+} \mathbb{D}$, Puneet Gambhir ${ }^{2^{*}}$ (D) and Manjit Kaur Mohi ${ }^{3 \dagger}$ (D)
}

\begin{abstract}
Background: The aim of this study is to elucidate the spectrum of commonly encountered anomalies affecting fetal genito-urinary system (GUS) on fetal MRI and examine its utility in providing better morphological information resulting in improved diagnostic accuracy and in detecting additional malformations. The study also aims to highlight the promising role of fetal MRI in the detection and characterization of renal fusion anomalies like the horseshoe kidney or developmental abnormalities such as renal agenesis/ectopia.

Results: The mean age of study participants was 29 years \pm 3 years. The gestation age of pregnant mothers ranged from 18 weeks and 1 day to 39 weeks and 0 day. Amniotic fluid was reduced or absent in $41 \%(N=13)$ and normal in $59 \%(N=18)$ of participating mothers. Overall, urinary obstruction was the commonest anomaly encountered (29\%) followed by the multicystic dysplastic kidney (MCDK) (22\%). Bilateral renal disease was seen in all mothers having features of anhydramnios $\{B / L$ MCDK $(N=3)$, autosomal recessive polycystic kidney disease (ARPKD) $(N=2)$, posterior urethral valves (PUV) $(N=2), B / L$ renal agenesis $(N=3)$, and megacystis $(N=1)\}$. Fusion anomalies (horseshoe kidney) and rotation anomaly (malrotation) were detected in one case each. Additional extrarenal findings were seen on fetal MRI in 35\% ( $N=11)$ cases.

Conclusions: Fetal MRI improves diagnostic accuracy in anomalies affecting the fetal kidney and genito-urinary systems by better morphological delineation. It has the ability to detect additional extra-renal malformations and perform a more accurate assessment of associated pulmonary hypoplasia. The diffusion-weighted sequence is particularly useful in confirming the diagnosis of renal agenesis/ectopia.
\end{abstract}

Keywords: Congenital malformations, Fetal MRI, Genito-urinary anomalies, Genito-urinary system, Renal anomalies

\section{Background}

Genito-urinary system (GUS) anomalies constitute an important and relatively common subset of fetal anomalies encountered in routine antenatal practice, comprising approximately $30-50 \%$ of all malformations detected on prenatal scans or at birth $[1,2]$. They exhibit a wide range of severity ranging from simple pyelectasis to

\footnotetext{
* Correspondence: drpuneetgmc@gmail.com

†'Saryu Gupta, Jaswinder Kaur Mohi and Manjit Kaur Mohi contributed equally to this work.

${ }^{2}$ Department of Community Medicine, Government Medical College and Rajindra Hospital, Patiala, Punjab 147005, India

Full list of author information is available at the end of the article
}

conditions incompatible with life such as bilateral renal agenesis. In addition, urinary tract disease has the potential to impact fetal lung maturity which in turn has important prognostic implications. An accurate diagnosis of these anomalies is therefore of paramount importance and can prove to be valuable in formulating management strategies and influencing parental counseling [3].

Antenatal ultrasound is invariably the initial screening modality for fetal anomaly scan and has proven ability in the detection of most malformations, but occasionally, it is hampered by lack of amniotic fluid leading to inconclusive or diagnostically inadequate examinations. Besides this, other technical limitations like maternal 
obesity and fetal pelvic bone ossification can also compromise the diagnostic potential of ultrasound in GU anomalies. Fetal MRI on the other hand is not significantly influenced by a lack of amniotic fluid or maternal obesity and therefore proves invaluable in establishing an accurate diagnosis in such cases. This study has been undertaken to evaluate the role of fetal MRI in improving the accuracy in the diagnosis of various fetal genitourinary system malformations and to examine its role in the detection of renal fusion anomalies like horseshoe kidney or developmental abnormalities such as renal agenesis/ectopia.

\section{Methods}

This is a prospective, observational, cross-sectional, and single-institution study conducted jointly by Departments of Radiodiagnosis and Obstetrics \& Gynaecology of our tertiary level institute. The study is of 1-year duration (March 6, 2019 to March 5, 2020) and was conducted after obtaining approval of the institutional ethics committee. A total of 31 consenting pregnant mothers having singleton fetuses with suspected anomalies pertaining to GUS diagnosed on a previous ultrasound and having gestation age $>18$ weeks were enrolled in the study.

\section{Technical considerations and institutional protocol Fetal MRI technique}

MRI was performed on $1.5 \mathrm{~T}$ platform (Siemens MAGNETOM Aera) using an 18 channel-phased array torso coil and head first approach. The mother was positioned in the supine or left lateral posture (in cases of advanced gestation to avoid IVC compression). No maternal sedation was given, and no intravenous contrast agent was administered.

Initial localizer was acquired with respect to a maternal axis in orthogonal planes using an ultrafast T2weighted sequence for purpose of fetus localization and pathology identification. The region of interest was kept in the center of the coil for optimum image quality. Subsequent image acquisition was done with respect to fetal imaging planes. The following sequences were routinely acquired in all patients using standard parameters:

1. T2 half Fourier-acquired single-shot turbo spinecho (HASTE) in axial, coronal, and sagittal planes adjusted to a fetal axis

2. True fast imaging with steady precession (TrueFISP) in at least one plane (preferably coronal plane)

3. T1 volumetric interpolated breath-hold examination (VIBE) and turbo fast gradient-echo sequence with a low-flip-angle shot (turbo-FLASH) in at least one plane (preferably axial)
4. Diffusion-weighted imaging (DWI) ( $b$ value 400 $700 \mathrm{~s} / \mathrm{mm}^{2}$ ) in an axial plane including both the fetal and maternal kidneys.

Alternate interleaved slices were acquired to reduce cross-talk while planning sequences with thinner or contiguous slices. Sequences were repeated when the image quality was degraded by fetal motion. Image stacks were transferred to advanced workstation and interpretation was done by two radiologists with 20 years and 30 years of experience respectively in fetal imaging. The final report was always given by consensus. Both the radiologists were blinded to the antenatal ultrasound diagnosis.

The reference gold standard for diagnosis in all surviving fetuses having a non-lethal malformation was a postnatal radiological investigation (ultrasound/MRI/cystouretherography) while in lethal malformations, an autopsy was the reference standard (under parental consent). In cases where consent was not obtainable for autopsy due to religious reasons, MRI diagnosis was considered as final.

\section{Results}

There were a total of 31 participants in our study group with a mean age of 29 years \pm 3 years. The youngest member was 21 years, and the eldest was 35 years of age. The gestation ages of examined fetuses ranged from 18 weeks and 1 day to 39 weeks and 0 day. Amniotic fluid was reduced (anhydramnios/oligohydramnios) in 41\% ( $N$ $=13)$ and was normal in $59 \%(N=18)$ of participating mothers. All mothers who showed evidence of anhydramnios $(N=11)$ had bilateral renal disease in form of $\operatorname{MCDK}(N=3)$, ARPKD $(N=2)$, PUV causing bilateral hydroureteronephrosis $(N=2)$, bilateral renal agenesis $(N=3)$, and megacystis secondary to urethral atresia $(N$ $=1)$. In many cases, MRI picked up additional findings which ranged from pulmonary hypoplasia, nuchal hygroma, pleural effusion, and placenta previa. The spectrum of various genito-urinary anomalies encountered along with associated findings detected is summarized in Table 1.

\section{Discussion}

In recent years, MRI has come to play an increasingly important role in fetal anomaly scanning. Its domain has expanded from being an adjunctive modality to one which is poised to become integral to fetal imaging protocols. This is because of several advantages like the superior soft-tissue contrast, development of ultrafast sequences, and ability to overcome the limitations of ultrasound like a poor sonic window in maternal obesity, oligohydramnios, and bony ossification [4-6].

The initial assessment of the fetal genito-urinary system is invariably performed by ultrasound. Unfortunately, 


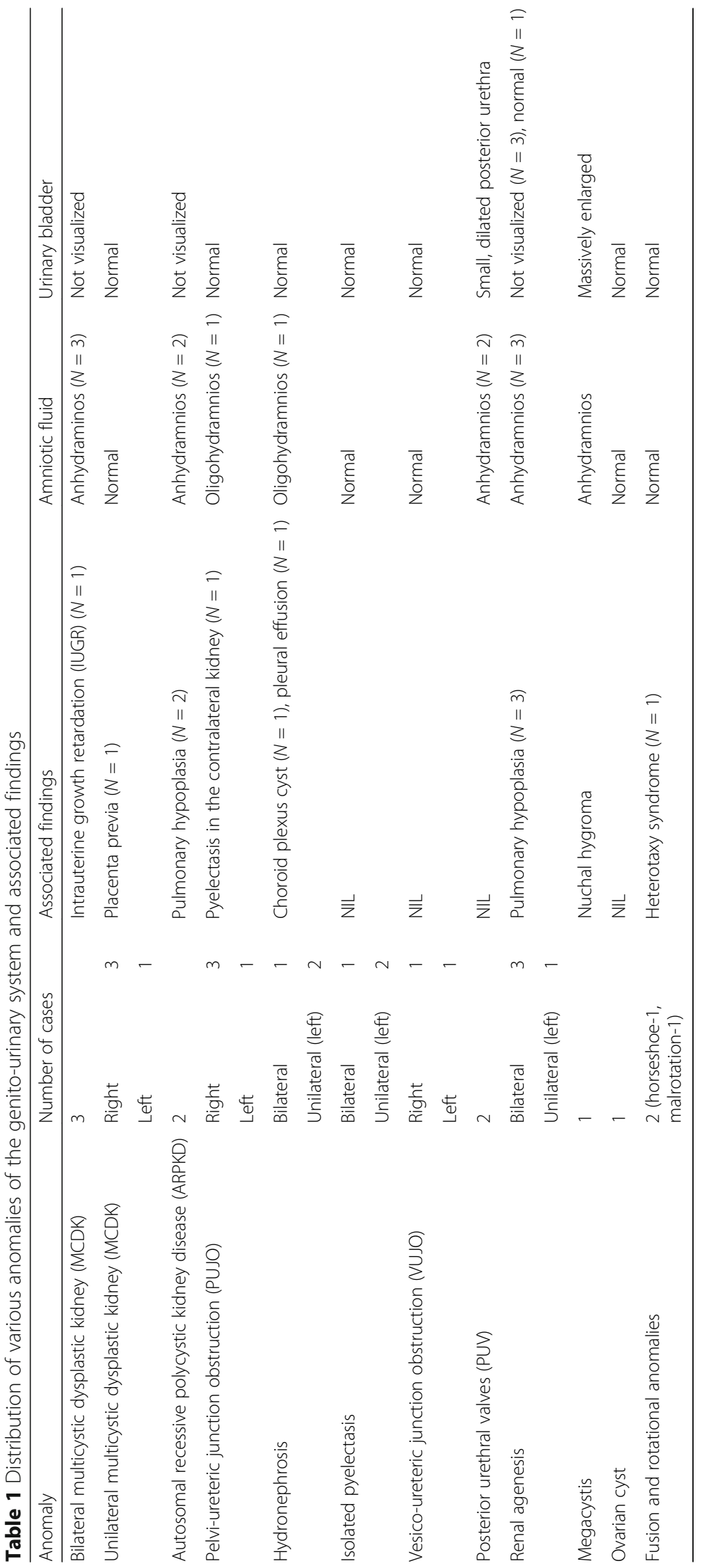


ultrasound as a standalone modality may be inadequate or inconclusive in many of the fetuses harboring these anomalies due to a frequent association of oligohydramnios or anhydramnios [7].

Thus, further assessment by fetal MRI is warranted in such cases for a more complete and informative anomaly evaluation. Ultrafast T2W sequence like HASTE has long been considered as the mainstay of genito-urinary system imaging on fetal MRI as it is relatively resistant to fetal motion due to sub-second slice acquisition and also provides exquisitely detailed images of fetal anatomy with high contrast and resolution. In this sequence, fetal kidneys are visualized as elliptical structures in a paravertebral location having an intermediate signal intensity. The renal cortex displays a slightly hypointense signal compared to the medulla, and the signal intensity ratio of the renal cortex to the medulla undergoes a steady increase with the advancement of gestational age $[8,9]$. The pelvicalyceal system and urinary tract exhibit hyperintense T2 signal intensity similar to the fluid. Balanced steady state-free precession sequences such as true-FISP also provide a comparable image quality with lesser radiofrequency heat generation. The inclusion of this sequence is useful in the delineation of GU anomalies which show obstructive changes. T1-weighted sequences like VIBE and faster versions like turbo FLASH are acquired as an add-on sequence in fetal GU imaging protocols. They are plagued by lower spatial resolution and susceptibility to fetal motion, but nonetheless have an undeniable complementary value for evaluation of fetal gastrointestinal tract, liver, and in the detection of hemorrhage/calcification. T1W images are particularly useful in differentiating dilated ureters from surrounding gut loops as meconium-filled large bowel is hyperintense compared to the hypointense ureter [9].

DWI sequence is an important component of fetal MRI GU imaging protocols in many institutions. Owing to the high water content of fetal organs, the echoplanar
DWI images are acquired at low $b$ values of $400-700$ to attain a better contrast. DWI sequences are particularly useful in the detection of renal parenchyma and assessment of its functionality. The normal fetal kidneys show restricted diffusion owing to the high cellularity resulting in a high DWI signal. This sequence is useful in making a confidant diagnosis of renal developmental abnormalities like renal agenesis or ectopia. An empty renal fossa in combination with absent DWI bright signal elsewhere favors agenesis while empty renal fossa with extrarenal/ pelvic location of bright signal points to ectopia. A diseased or pathological fetal kidney has decreased cellularity and will therefore show reduced DWI signal brightness. This property can be exploited for prediction of renal function by a comparative feto-maternal renal apparent diffusion coefficient (ADC) map value evaluation with increased ADC values indicative of impaired renal functions.

MRI is also useful in the assessment of any associated anorectal abnormalities which can occur as part of VATER (vertebral, anal atresia, trachea-esophageal fistula, renal anomalies syndrome). The various genital system diseases such as ovarian cysts or diseases affecting perineal structures like cloacal exstrophy can be also better characterized by this modality.

For discussion purpose, the anomalies of the fetal genito-urinary system encountered in our study have been categorized into broad aetiological groups as follows:

\section{Cystic renal disease}

This group comprises conditions like multi-cystic dysplastic kidney (MCDK) or simple cysts of kidneys. MCDK may be sometimes difficult to differentiate from hydronephrosis on ultrasound. On MRI however, owing to its superior soft-tissue contrast, the disease morphology is better seen. In MCDK renal parenchyma is absent consequent to the aberrant ureteral bud

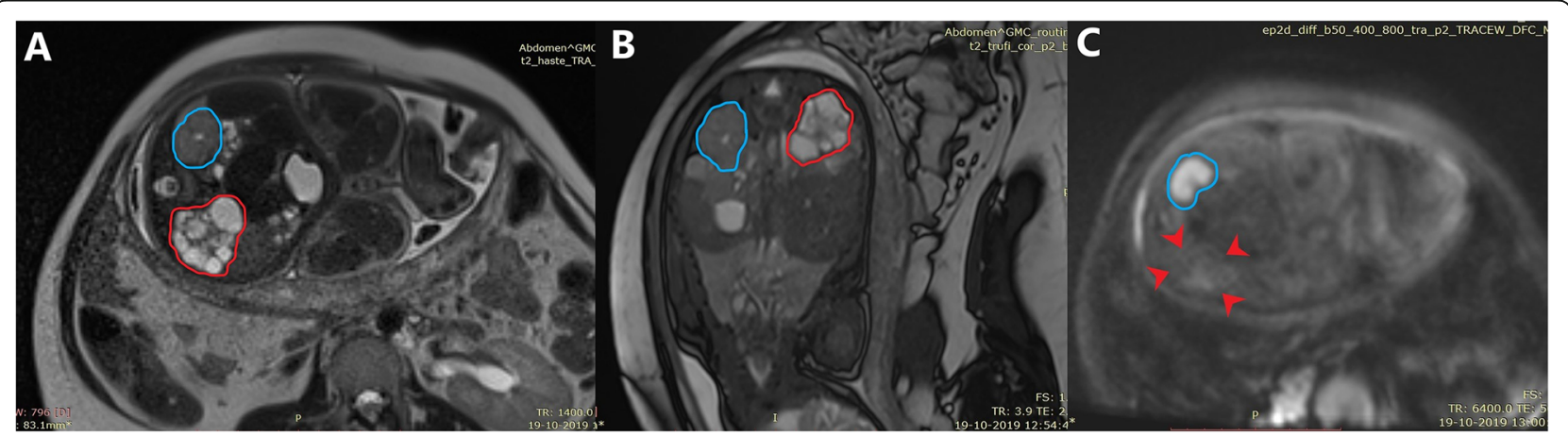

Fig. 1 Right-sided multicystic dysplastic kidney disease: maternal age: 25 years; period of gestation: 38 weeks 1 day; T2 HASTE axial (a) and T2 TRUFI coronal (b): Left kidney is normal (blue tracing) while right kidney (red tracing) displays lobulated contour with multiple, variable-sized, non-communicating and clustered internal cysts. DWI axial (c) shows a low signal in the right kidney (arrowheads) with normal bright signal in left kidney 


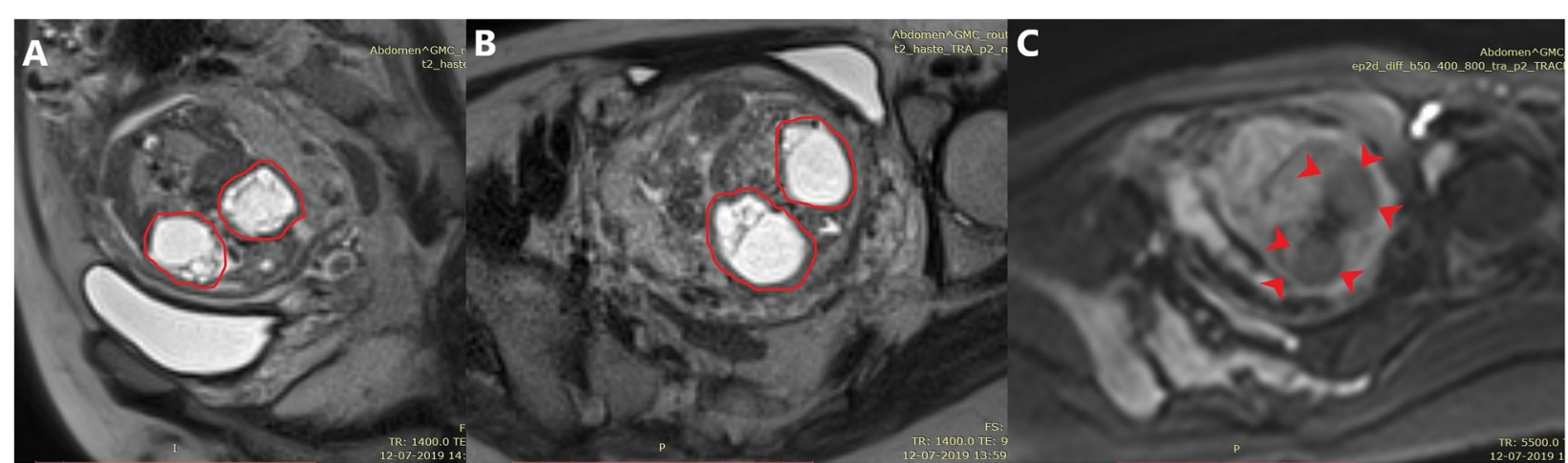

Fig. 2 Bilateral multicystic dysplastic kidney disease: maternal age: 20 years; period of gestation: 21 weeks 2 days: T2 HASTE coronal (a) and axial (b) images show multicystic dysplastic changes involving both kidneys (red tracing) with associated anhydramnios. Axial DWI (c) shows loss of normal hyperintense signal in both kidneys (arrowheads)

development and is replaced by multiple peripheral and central non-communicating cysts distorting the renal shape (Fig. 1a, b and Fig. 2a, b). The lack of identifiable renal parenchyma results in low signal on DWI images which can be a useful pointer for differentiation between the two entities (Figs. 1c and 2c). In our study, we encountered 7 cases of MCDK (unilateral in 4 and bilateral in 3 cases). All the bilateral cases expectedly showed evidence of anhydramnios and pulmonary hypoplasia. We found MRI to be especially useful in evaluating bilateral MCDK cases as the associated lack of amniotic had compromised the sonic window. Our observation that MRI is helpful in differentiating MCDK from hydronephrosis is in consonance with similar observations recorded in earlier studies $[1,5,10,11]$. According to these studies, MRI is helpful in displaying the disease morphology in a better manner, and in MCDK, it shows multiple variable-sized non-communicating cortical and medullary cysts having hyperintense T2 signal and low DWI signal. In case of hydronephrosis, the dilated renal pelvis shows a more central location, and intercommunication between the dilated calyces is also better appreciated allowing for a differentiation between the two entities.

In one rare case of a solitary cystic variant of MCDK seen in our study, MRI proved helpful in distinguishing the MCDK cyst from pelviureteric junction obstruction (PUJO). The contralateral kidney however showed typical MCDK features with a multicystic appearance.

\section{Autosomal recessive polycystic renal disease (ARPKD)}

ARPKD is characteristic by marked symmetric nephromegaly with maintained reniform shape and diffusely increased T2 signal intensity which occurs due to increased water content of renal parenchyma. Usually,

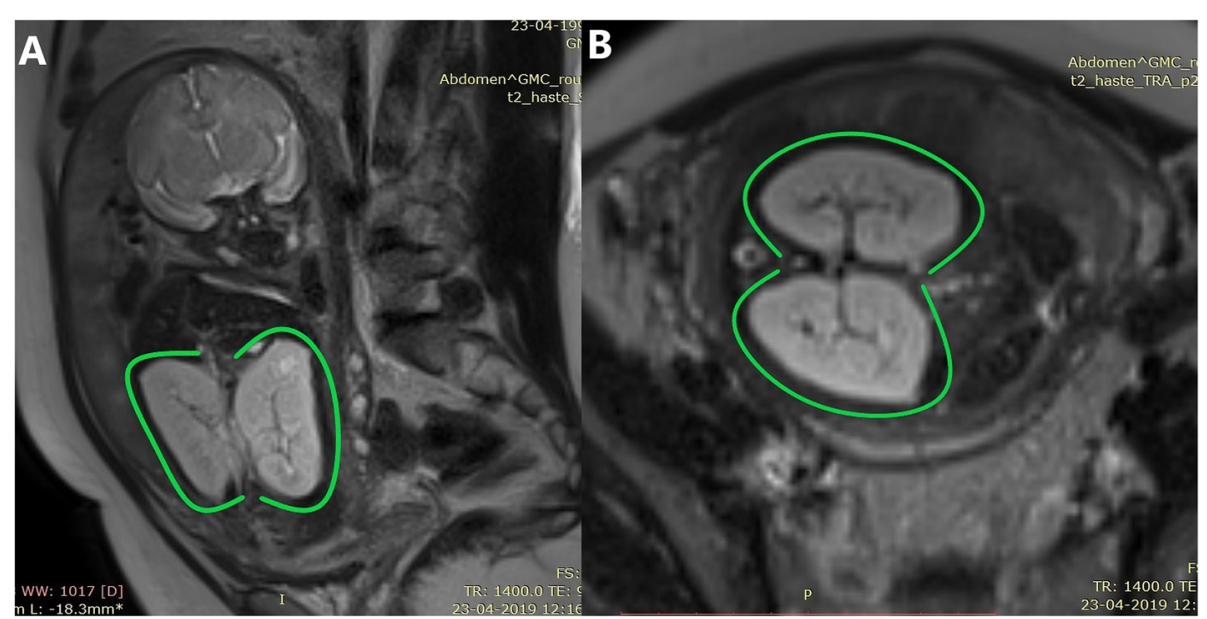

Fig. 3 Bilateral autosomal recessive polycystic kidney disease: maternal age: 27 years; period of gestation: 32 weeks 6 days; T2 HASTE coronal (a) and axial (b): nephromegaly with diffusely increased signal on fluid-sensitive sequences involving both the right and left kidneys (green arcs); the urinary bladder was not visualized (not shown); there was also evidence of anhydramnios and bilateral pulmonary hypoplasia 


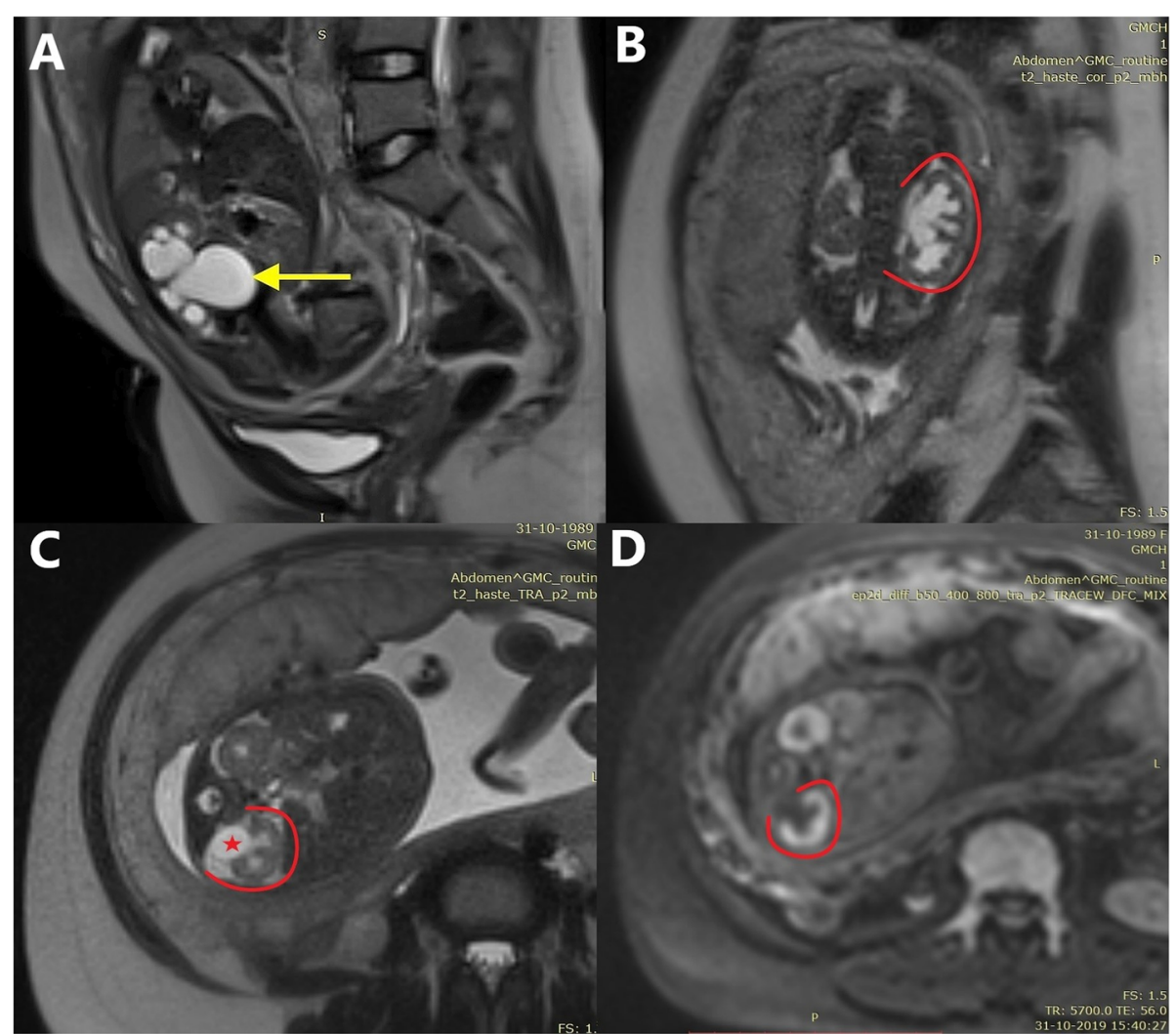

Fig. 4 a Pelviureteric junction obstruction (PUJO): maternal age: 22 years; period of gestation: 32 weeks 2 days: T2 HASTE (a): the right kidney shows dilatation of the pelvicalyceal system with pelviectasis out of proportion to caliectasis and non-visualization of the ipsilateral ureter consistent with PUJO (yellow arrow); b, c Unilateral hydronephrosis: T2 HASTE coronal (b) and axial (c) images show moderately dilated pelvicalyceal system (red arc) with maintained bright signal on DWI (d). Note the posteriorly directed renal pelvis (asterisk) suggestive of malrotation

cysts are not visualized in ARPKD but sometimes few tiny cysts (not exceeding $1-2 \mathrm{~cm}$ in size) may be present. In bilateral cases, the urinary bladder is frequently not visualized [12] and amniotic fluid is often reduced manifesting as oligohydramnios or anhydramnios. We came across 2 cases of bilateral ARPKD in our study with typical MRI imaging findings of nephromegaly with high T2 signal (Fig. 3a, b). In both cases, associated findings of anhydramnios and lung hypoplasia were present

\section{Urinary tract dilatation}

Urinary tract dilatation is one of the commonest entities seen in fetuses with GUS anomalies with mild pelviectasis at the least severe end of the spectrum. Of the multitude of causes resulting in urinary obstruction, PUJO is the most frequent with an incidence of approximately $35 \%$ [7], followed by uretero-pelvic junction obstruction (UPJO) with an incidence of close to $10 \%$. Other notable causes of fetal urinary tract dilatation include vesicoureteric reflux (VUR) disease, ureterocele (mostly in association of duplication anomaly), posterior urethral valves (PUV) anomaly, urethral atresia, and megacystis microcolon hypoperistalsis syndrome. In our study too, anomalies resulting in urinary tract dilatation were the most frequent, comprising $29 \%$ of the total GUS anomalies encountered, and among these, PUJO was the most common entity (Fig. 4a) followed by HDN (Fig. 4b-d).

We found MRI to be very useful in delineating confidently the level of obstruction as the collecting system upstream to this level gets dilated and shows a hyperintense signal on FFE and ultrafast T2 sequences, thus making it more conspicuous. Additionally, MRI was useful in assessing pelvic anatomy particularly in cases of lower urinary tract obstruction. The typical keyhole appearance of the dilated posterior urethra was also much better appreciated on MRI (Fig. 5a-d). Recent studies too have espoused the benefits of fetal MR in better pelvic anatomical delineation. They also observed that owing to an excellent spatial resolution of fetal MRI and non-affection of image quality by fetal pelvic bone ossification, MRI allows a better and more accurate identification of perineal structures and pelvic anatomical landmarks like the rectum, bladder, vagina, and uterus. $\mathrm{T} 1$ hyperintense signal of meconium laden rectum is 


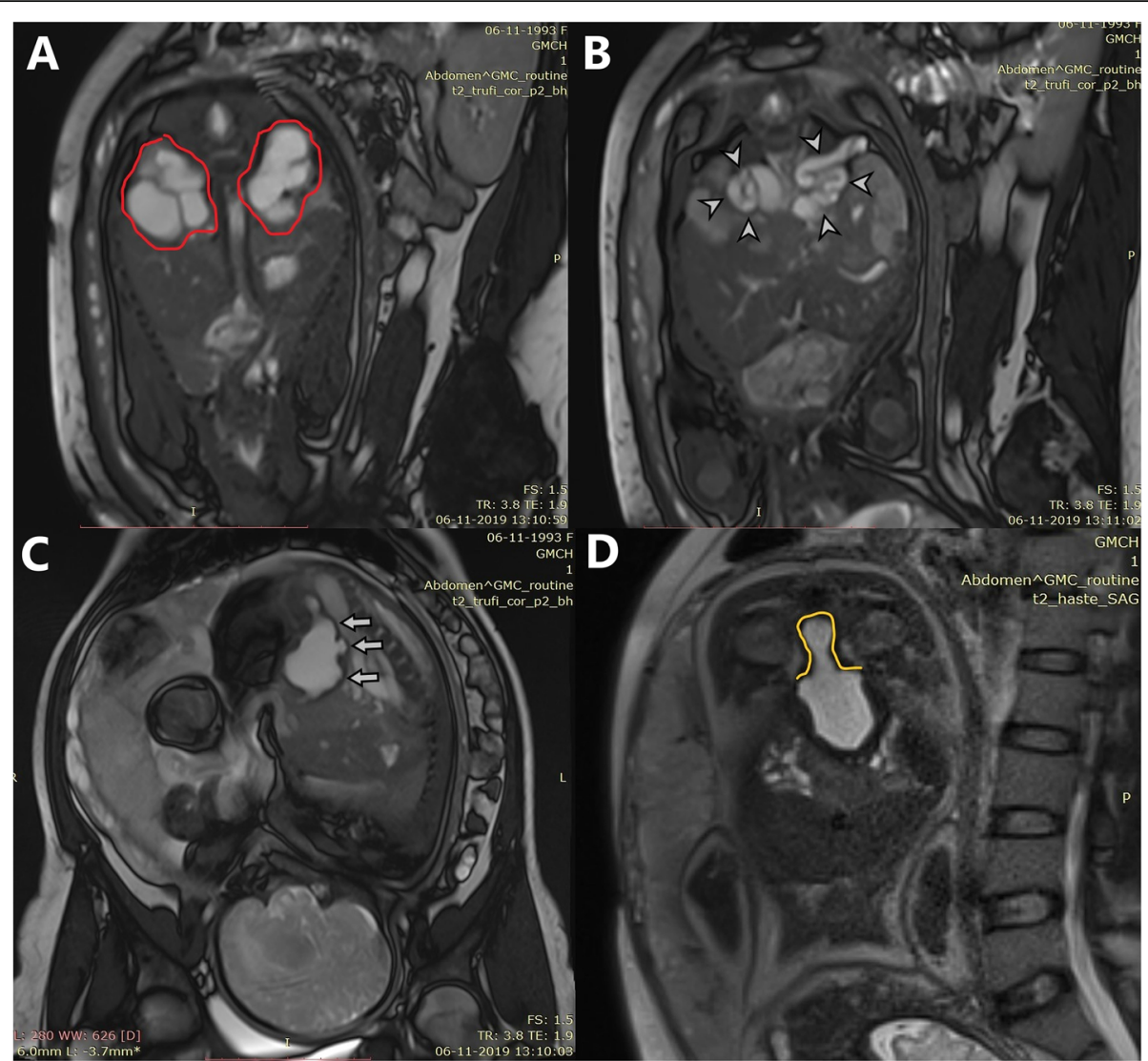

Fig. 5 Posterior urethral valves: maternal age: 26 years; period of gestation: 39 weeks 0 days; coronal T2 TRUFI images (a, b) showing gross bilateral hydroureteronephrosis (red tracing in $\mathbf{a}$ and arrowheads in $\mathbf{b}$ ). T2 TRUFI sagittal (c) showing trabeculated bladder outline and the T2 HASTE Coronal image (d) showing "typical key hole appearance" of the dilated posterior urethra (yellow trace). There is also evidence of anhydramnios

particularly useful in assessing the inter-relationship of pelvic organs with each other which is pivotal for diagnosing complex genito-urinary anomalies. Cloacal abnormalities, ectopic ureteral insertion in renal duplication anomalies, and posterior urethral dilatation are some of the anomalies which are better diagnosed $[1,5,6,13-15]$.

MRI can also be utilized for providing additional useful information on some other renal features like cortical thickness and signal intensity of renal parenchyma. Severe hydronephrosis results in cortical thinning whereas increased parenchymal T2 signal intensity may be seen in dysplastic changes $[10,11,16]$.

\section{Fusion anomaly}

Prenatal diagnosis of fusion anomaly like the horseshoe kidney is a rare occurrence. We diagnosed a case of fetal horseshoe kidney in our study which was missed altogether on ultrasound (Fig. 6a-c). Additionally, the left moiety of the horseshoe kidney showed dysplastic changes with small cysts; however, the ureters were normal caliber in our case. It is recommended that in the horseshoe kidney, ureters should routinely be assessed for any anomalous course or dilatation [4].

\section{Renal agenesis}

MRI can play a very important role in the evaluation of renal agenesis especially if it is bilateral. In such cases, there is invariably severe oligohydramnios or anhydramnios, and assessment by ultrasound is unreliable [17]. DWI can be particularly useful in the demonstration of missing bright signal in renal fossa [18]. We had four cases of renal agenesis (three of bilateral and one of unilateral) and found DWI to be a helpful sequence in increasing the diagnostic confidence (Fig. 7a-d). Pulmonary hypoplasia is a frequent accompaniment of bilateral agenesis and can be assessed more accurately on MRI. This in turn has important implications on fetal prognosis.

\section{Ovarian cyst}

An ovarian cyst is a relatively common intra-abdominal mass in female fetuses, and MRI is indicated mainly to clarify anatomy, provide additional diagnostic details like intracystic hemorrhage or occurrence of complex 


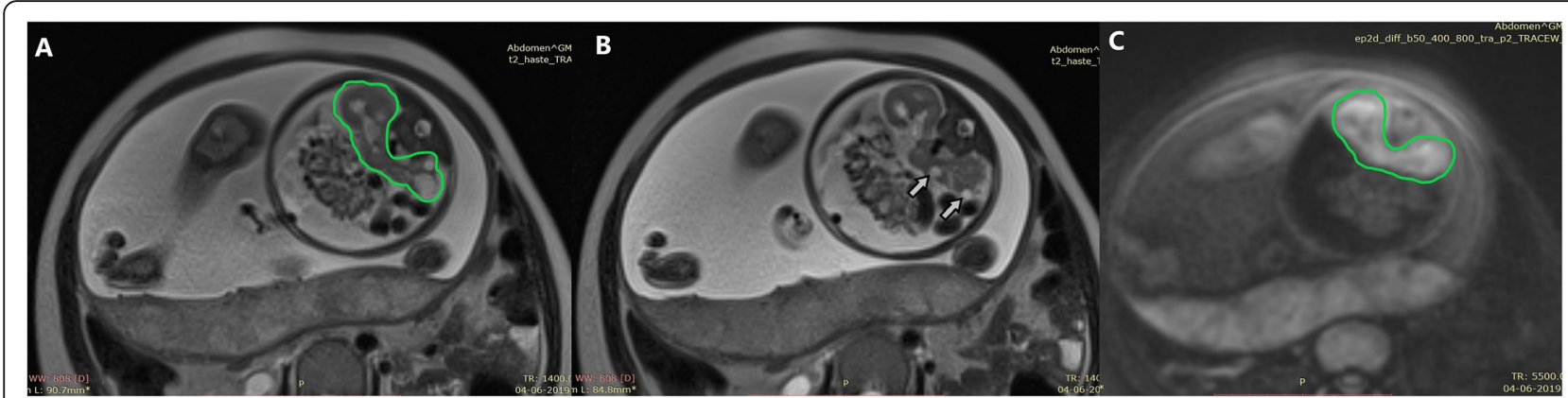

Fig. 6 The horseshoe kidney in a case of heterotaxy syndrome: maternal age: 26 years; period of gestation: 30 weeks; T2 HASTE axial (a, b) and DWI axial (c) images show typical horseshoe configuration of the fetal kidney (green tracing) with small dysplastic cysts (arrows)

changes. The T1-weighted sequences are useful in detecting bleed as it will give increased signal intensity even in dilution. We had one case of unilocular ovarian cyst (Fig. 8a-b) which remained uncomplicated throughout the pregnancy but underwent torsion leading to oophorectomy a few days after birth. Serial ultrasounds are always recommended whenever an ovarian cyst is diagnosed prenatally. Monitoring of cyst size is considered important from a treatment point of view since cysts smaller than $50 \mathrm{~mm}$ may regress and are therefore are kept on observation whereas cysts greater than 50 $\mathrm{mm}$ are unlikely to regress, prone for torsion and may therefore be treated by in utero or post-delivery aspiration $[19,20]$.

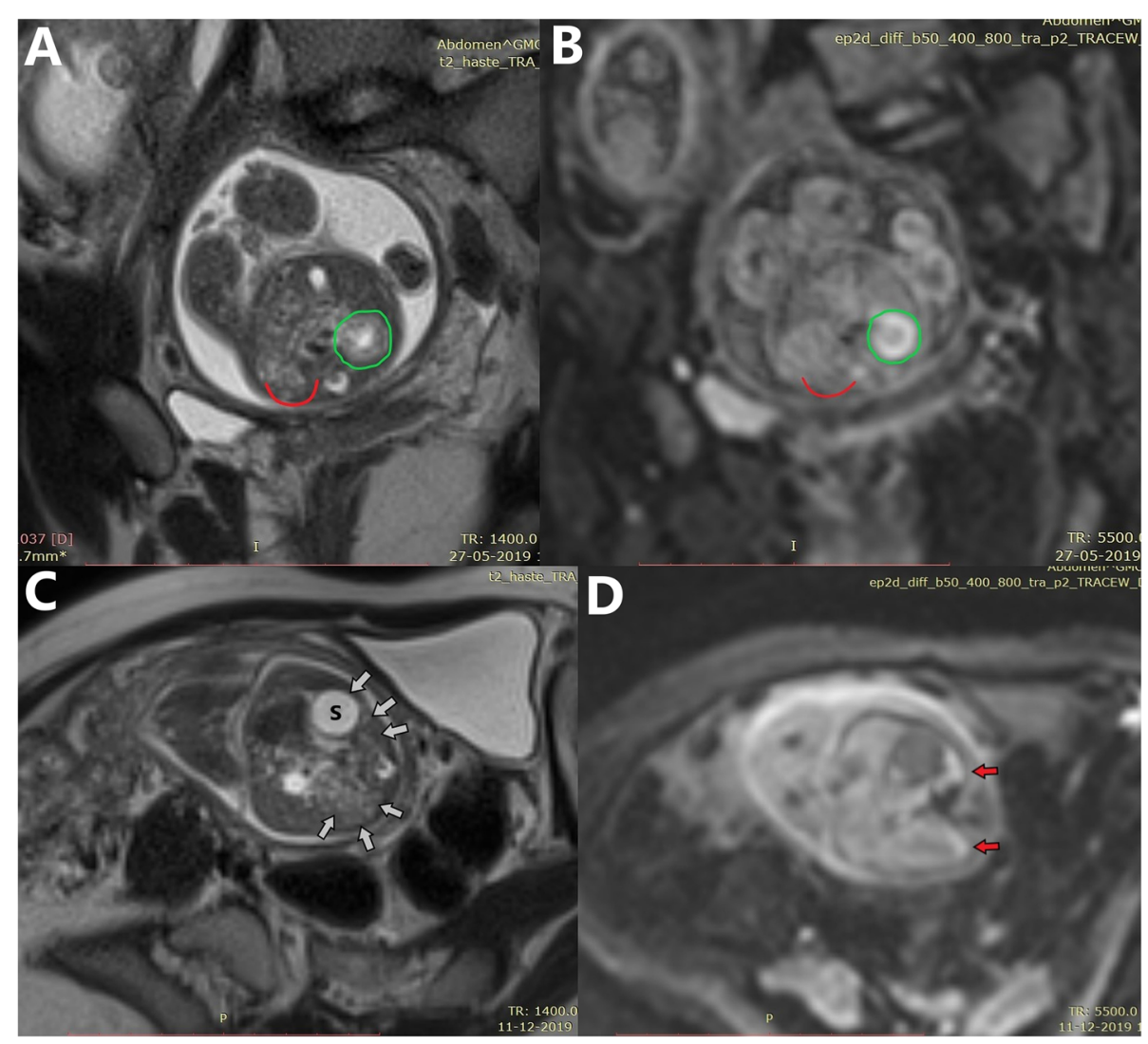

Fig. 7 a, b Unilateral renal agenesis: maternal age: 25 years; period of gestation: 24 weeks 2 days; T2 HASTE axial (a) and DWI axial (b) show one normal fetal kidney with bright DWI signal (green tracing) while the on contralateral side the renal fossa is empty and bright DWI signal is missing (red arc). c, d Bilateral renal agenesis: maternal age: 21 years; period of gestation: 25 weeks 4 days; T2 HASTE axial (c) and DWI axial (d): bilateral renal fossae are empty (arrows) with absent DWI bright signal in bilateral renal fossae (red arrows); urinary bladder was not visualized. There is also evidence of anhydramnios and bilateral pulmonary hypoplasia 


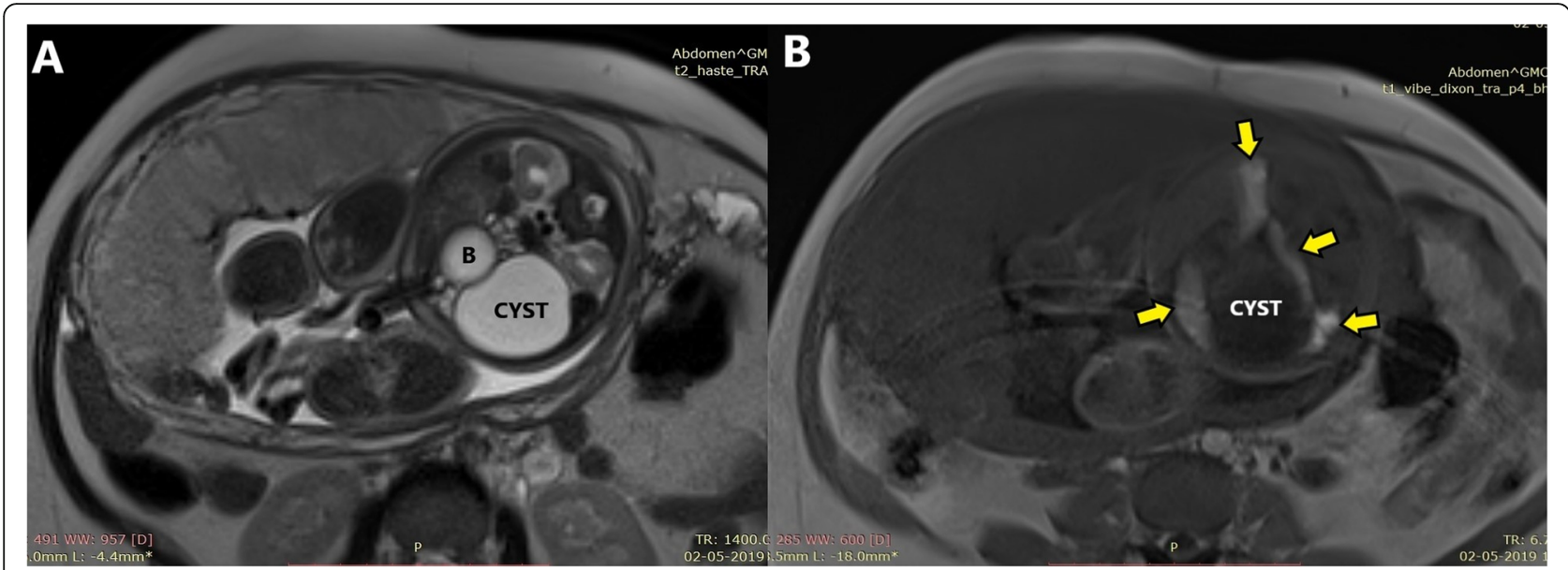

Fig. 8 Ovarian cyst: maternal age: 24 years; period of gestation: 35 weeks 2 days: T2 HASTE axial (a) a simple ovarian cyst in the left abdominopelvic region (labeled "cyst") adjacent to the bladder (labeled as b) with no features of complications. Axial T1 VIBE image (b) clarifies the inter-relationship of cyst with adjacent $\mathrm{T} 1$ hyperintense meconium-filled colon loops

Limitations of our study were the selection bias that occurred because the study sample comprised of cases of fetal GUS anomalies already diagnosed or suspected on ultrasound. Another limitation was the lack of depiction of a complete spectrum of GU system anomalies mainly because of shorter study duration, low prevalence rates, and focus on one system only.

\section{Conclusion}

Fetal MRI plays an important role in a confident assessment of anomalies affecting genito-urinary system. It overcomes the obstacles posed by a lack of amniotic fluid and has added value in terms of refining, modifying, or adding to the diagnostic information provided by ultrasound. It has a promising role in the detection of renal fusion anomalies like the horseshoe kidney or developmental abnormalities such as renal agenesis/ectopia and may detect additional abnormalities pointing to an underlying syndromal etiology or give added information on extrarenal organ systems like lung hypoplasia. It also has the potential to guide management strategies and influence parental counseling. In many non-lethal anomalies of GUS, accurate and timely diagnosis by fetal MRI can result in the institution of appropriate therapeutic intervention which can translate into improved survival benefit and better quality of post-natal life. Fetal MRI is hence recommended in the diagnostic workup of genitourinary tract anomalies whenever ultrasound is noninformative or equivocal.

\section{Abbreviations}

GUS: Genito-urinary system; MCDK: Multicystic dysplastic kidney; ARPK D: Autosomal recessive polycystic kidney disease; PUV: Posterior urethral valves; HASTE: Half Fourier single-shot turbo spin-echo; True-FISP: True fast imaging with steady state-free precession; VIBE: Volume interpolated breathhold examination; Turbo-FLASH: Turbo fast-gradient echo sequence with low-flip-angle shot; DWI: Diffusion-weighted imaging; FLASH: Fast low-angle shot; IUGR: Intrauterine growth retardation; PUJO: Pelviureteral junction obstruction; VUJO: Vesicoureteral junction obstruction; ADC: Apparent diffusion coefficient; VATER: Vertebral, anal atresia, trachea-esophageal fistula, renal anomalies

\section{Acknowledgements}

Mr. Jarnail Singh, Radiographer MRI Section, for his sincerity, dedication, and hardwork during his involvement in this research work.

\section{Authors' contributions}

SG made a substantial contribution to the concepts of the study, image acquisition, interpretation, and drafting of the manuscript. JKM made substantial contributions to the design of the work and the acquisition, analysis, and OR interpretation of data and substantively revised the manuscript. PG made substantial contributions to the conception and design of the work, and analysis and OR interpretation of data and drafted the work. MKM made substantial contributions to the design of the work and substantively revised it. The authors read and approved the final manuscript.

\section{Funding}

Source of funding-NIL

\section{Availability of data and materials}

The datasets used and/or analyzed during the current study are available from the corresponding author on reasonable request.

\section{Ethics approval and consent to participate}

Due approval has been obtained from the institutional ethics committee of the Government Medical College_-Patiala, for carrying out this study vide committee approval No.TRG.9(310)2019/2235 dated March 6, 2019. Written informed consent was obtained from all the pregnant mothers for their voluntary participation in the study in their vernacular language as well as in English.

\section{Consent for publication}

All participants included in this research gave written informed consent to publish the data contained within this study.

\section{Competing interests \\ None}

\section{Author details}

'Department of Radiodiagnosis, Government Medical College and Rajindra Hospital, Patiala, Punjab, India. ${ }^{2}$ Department of Community Medicine, Government Medical College and Rajindra Hospital, Patiala, Punjab 147005, 
India. ${ }^{3}$ Department of Obstetrics and Gynaecology, Government Medical College and Rajindra Hospital, Patiala, Punjab, India.

Received: 30 April 2020 Accepted: 4 August 2020

Published online: 18 August 2020

\section{References}

1. Gomez Huertas M, Culianez Casas M, Molina Garcia FS, Carrillo Badillo MP, Pastor Pons E (2016) Complementary role of magnetic resonance imaging in the study of the fetal urinary system. Radiologia 58:101-110

2. Behairy NHED, El Din LAS, Hanoun NMF, El Raof MA, Ali MAEK (2015) Diagnostic value of fetal MRI in evaluating fetal urinary anomalies. Egypt J Radiol Nuclear Med 46:521-528

3. Fazecas TM, Araujo Junior E, Werner $H$, Daltro P, Peixoto AB, Lima GM et al (2019) Applicability of magnetic resonance imaging in the assessment of fetal urinary tract malformations. Can Assoc Radiol J 70:83-89

4. Saleem SN (2014) Fetal MRI: an approach to practice: a review. J Adv Res: 507-523

5. Faghihimehr A, Gharavi M, Mancuso GSG (2018) Fetal MR imaging in urogenital system anomalies. J Matern Fetal Neonatal Med 32:3487-3494

6. Millischer AE, Grevent D, Rousseau V, O'Gorman N, Sonigo P, Bessieres B et al (2017) Fetal MRI compared with ultrasound for the diagnosis of obstructive genital malformations. Prenat Diagn 37:1138-1145

7. Hörmann M, Brugger PC, Balassy C, Witzani L, Prayer D (2006) Fetal MRI of the urinary system. Eur J Radiol 57:303-311

8. Chalouhi GE, Millischer AÉ, Mahallati H, Siauve N, Melbourne A, Grevent D et al (2020) The use of fetal MRI for renal and urogenital tract anomalies. Prenat Diagn 40:100-109

9. Furey EA, Bailey AA, Twickler DM (2016) Fetal MR imaging of gastrointestinal abnormalities. Radiographics 36:904-917

10. Caire JT, Ramus RM, Magee KP, Fullington BK, Ewalt DH, Twickler DM (2003) MRI of fetal genitourinary anomalies. AJR Am J Roentgenol 181:1381-1385

11. Amin RS, Nikolaidis P, Kawashima A, Kramer LA, Ernst RD (1999) Normal anatomy of the fetus at MR imaging. Radiographics 19:S201-S214

12. Liu YP, Cheng SJ, Shih SL, Huang JK (2006) Autosomal recessive polycystic kidney disease: appearance on fetal MRI. Pediatr Radiol 36:169

13. Mouriquand PDE, Whitten M, Pracros JP (2001) Pathophysiology, diagnosis and management of prenatal upper tract dilatation. Prenat Diagn 21:942951

14. Thomas DFM (1998) Prenatally detected uropathies: epidemiological considerations. Br J Urol 81(Suppl 2):8-12

15. James CA, Watson AR, Twining P, Rance CH (1998) Antenatally detected urinary tract abnormalities; changing incidence and management. Eur J Pediatr 157:508-511

16. Shinmoto H, Kashima K, Yuasa $Y$, Tanimoto A, Morikawa $Y$, Ishimoto $H$ et al (2000) MR imaging of non-CNS fetal abnormalities: a pictorial essay. Radiographics 20:1227-1243

17. Bronshtein M, Amit A, Archiron R, Noy I, Blumenfield Z (1994) The early prenatal sonographic diagnosis of renal agenesis: techniques and possible pitfalls. Prenat Diagn 14:291-297

18. Brewerton LJ, Chari RS, Liang Y, Bhargava R (2005) Fetal lung-to-liver signal intensity ratio at MR imaging: development of a normal scale and possible role in predicting pulmonary hypoplasia in utero. Radiology 235:1005-1010

19. Trinh TW, Kennedy AM (2015) Fetal ovarian cysts: review of imaging spectrum, differential diagnosis, management, and outcome. Radiographics 35:621-635

20. Nemec U, Nemec SF, Bettelheim D, Brugger PC, Horcher E, Schöpf $V$ et al (2012) Ovarian cysts on prenatal MRI. Eur J Radiol 81:1937-1944

\section{Publisher's Note}

Springer Nature remains neutral with regard to jurisdictional claims in published maps and institutional affiliations.

\section{Submit your manuscript to a SpringerOpen ${ }^{\circ}$ journal and benefit from:}

- Convenient online submission

- Rigorous peer review

- Open access: articles freely available online

- High visibility within the field

- Retaining the copyright to your article

Submit your next manuscript at $\boldsymbol{\nabla}$ springeropen.com 